\title{
Kontrolle ist gut, Strafe noch besser?
}

\section{Zur neuen (?) Punitivität in der Sozialen Arbeit}

In den vergangenen Jahren wird eine zunehmende Kontroll- und Straforientierung in der Sozialen Arbeit diskutiert (vgl. Dollinger/ Schmidt-Semisch 2011, Kess1 2011, Lutz 2010, Oelkers/Ziegler 2009, Lautmann 2008). Dabei geht es nicht nur in verschiedenen Handlungskontexten Professioneller, sondern beispielsweise auch bei Studierenden in der Sozialen Arbeit, um eine verstärkte Tendenz $z u$,zero tolerance“-Strategien, aktivierungspädagogischen Methoden und punitiven Haltungen. Diskutiert wird die Frage, ob diese Punitivitätsorientierung tatsächlich eine neue Entwicklung darstellt, inwiefern es sich um einen Paradigmenwechsel im großen Stil handelt und ob der Diskurs um die Straforientierung möglicherweise ein bekanntes Phänomen in der Sozialen Arbeit darstellt (vgl. Kessl 2011, Dollinger 2011).

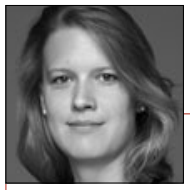

Nadia Kutscher *1972

Dr., Dipl. Päd., Dipl. Sozialpäd. (FH), Professorin für Soziale Arbeit und Ethik an der Universität Vechta. Arbeitsschwerpunkte: Mediatisierung der Sozialen Arbeit, Bildung und Ungleichheit, Normativität und Soziale Arbeit. nadia.kutscher@ uni-vechta.de
Loï Wacquant beschreibt in seinem Buch „Bestrafen der Armen. Zur neuen Regierung der sozialen Unsicherheit“ (2009) am Beispiel der USA mit Blick auf Entwicklungen in Europa Entwicklungen in der Wohlfahrts- und Strafverfolgungspolitik unter den Bedingungen neoliberaler Governance. Im Kontext politischer und gesellschaftlicher Ökonomisierungstendenzen, die auch den Wohlfahrtsstaat mit transformieren, wird dabei die Kontroll- und Straforientierung zum Aspekt der Effizienzsicherung wohlfahrtsstaatlicher Leistungen unter Unsicherheitsbedingungen. Andere Autoren stellen diese Entwicklungen in Zusammenhang mit einer eingeschränkteren Toleranz in der Gesellschaft gegenüber abweichendem Verhalten. Blickt man in die Geschichte Sozialer Arbeit, so findet sich hier allerdings eine lange Tradition straf- und kontrollorientierter Ansätze: von den Arbeits- und Zuchthäusern über die Kontrolle durch Armenpfleger im Elberfelder System bis hin zu heutigen Alg-II-Regelungen und aktivierungs- und „konfrontativ“ pädagogischen Konzepten, aber auch hinsichtlich der Moralisierung spezifischer Formen der Lebensführung (z.B. in der „Underclass“Debatte - vgl. u.a. Kessl/Reutlinger/Ziegler 2007) und deren pädagogische Bearbeitung bzw. beabsichtigte Überwindung.

Dieser Schwerpunkt nimmt die Diskussion um die Punitivität in der Sozialen Arbeit auf und betrachtet Fragen von Strafe und Kontrolle aus verschiedenen Perspektiven. Tilman Lutz thematisiert in seinem Beitrag den Zusammenhang zwischen aktivierendem Staat und Kontrollorientierung und fordert eine kritische Auseinandersetzung innerhalb der Profession und Disziplin Sozialer Arbeit mit diesen Entwicklungen. Bernd Dollinger zeigt in seinem Artikel die Widersprüchlichkeit und Brüchigkeit punitiver Aspekte. Er weist darauf hin, dass die strafende Perspektive in der Sozialen Arbeit nicht alle AdressatInnengruppen gleichermaßen fokussiert, sondern spezifische Personengruppen und Problemlagen besonders davon betroffen sind, so dass Soziale Arbeit hier eine gesellschaftli- che „Wächterfunktion“ mit Blick auf bestimmte Zielgruppen wahrnimmt. Nina Oelkers verweist auf eine damit verbundene mögliche „Entkollektivierung“ sozialer Probleme und zeigt anhand erster Ergebnisse von Studierendenbefragungen an verschiedenen Universitäten verbreitet Vorurteile gegenüber AdressatInnen und skeptische Haltungen gegenüber wohlfahrtsstaatlicher Versorgung unter den SozialarbeiterInnen in spe auf. Stefan Wellen setzt sich in seinem Beitrag mit Haltungen des Kulturrassismus gegenüber Langzeitarbeitslosen auseinander und wirft dabei die Frage nach der Rolle einer Sozialen Arbeit in diesem Zusammenhang auf, die als Teil der Verwaltung von „normal“ und ,anormal“ an der Reproduktion von diskriminierenden Unterscheidungen mitwirkt.

Die Beiträge diskutieren somit, inwiefern eine Kontroll- und Straforientierung in der Praxis Sozialer Arbeit zur Stigmatisierung gesellschaftlich „unerwünschter“, benachteiligter Gruppen beiträgt und Soziale Arbeit damit zur Akteurin politischer und gesellschaftlicher Ausgrenzungspolitiken wird.

\section{Literatur}

DOLLINGER, B. UND SCHMIDT-SEMISCH, H. (HRSG.) (2011).

Gerechte Ausgrenzung? Wohlfahrtsproduktion und

die neue Lust am Strafen. Wiesbaden.

KESSL, F. (2011).

Punitivität in der Sozialen Arbeit - von der Normalisierungszur Kontrollgesellschaft. IN: Dollinger, B. u. Schmidt-Semisch, H. (Hrsg.), Gerechte Ausgrenzung? (S. 131-143). Wiesbaden.

KESSL, F., REUTLINGER, C. U. ZIEGLER, H. (HRSG.) (2007).

Erziehung zur Armut? Soziale Arbeit und die ,neue Unterschicht'. Wiesbaden.

LAUTMANN, R. (2008).

„Gibt es nichts Wichtigeres?" - Sexualität, Ausschluss und Soziale

Arbeit. IN: Anhorn, R., Bettinger, F. u. Stehr, J. (Hrsg.), Sozialer

Ausschluss und Soziale Arbeit (S. 273-290). Wiesbaden

LUTZ, T. (2010)

Soziale Arbeit im Kontrolldiskurs: Jugendhilfe und ihre Akteure in postwohlfahrtstaatlichen Gesellschaften. Wiesbaden.

OELKERS, N. U. ZIEGLER, H. (2009).

Punitivität, Verantwortung und Soziale Arbeit. IN: Zeitschrift

für Jugendkriminalrecht und Jugendhilfe 1 (S. 38-44).

WACQUANT, L. (2009).

Bestrafen der Armen. Zur neuen Regierung der sozialen Unsicherheit. Opladen. 